\title{
Perfil Somatotípico del Futbolista Profesional Chileno
}

\author{
Somatotype Profile of Professional Male Soccer Chilean Players
}

Henríquez-Olguín, C. ; Báez, E. ${ }^{* *, * * * ;}$ Ramírez-Campillo, R.**** \& Cañas, R.*******

\begin{abstract}
HENRÍQUEZ-OLGUÍN, C.; BÁEZ, E.; RAMÍREZ-CAMPILLO, R. \& CAÑAS, R. Perfil somatotípico del futbolista profesional chileno. Int. J. Morphol., 31(1):225-230, 2013.

RESUMEN: El presente estudio pretende describir el somatotipo del futbolista profesional chileno, así como determinar la variabilidad existente en el somatotipo por posición de juego. Cien jugadores profesionales varones (edad 23 $\pm 4,4$ años), participantes del campeonato nacional de fútbol chileno fueron incluidos en el estudio. Se realizó la medición de 10 variables antropométricas según las normas de la International Society for the Advancement of Kinanthropometry (ISAK). Se calcularon los 3 componentes del somatotipo de Heath-Carter por cada posición de juego. El somatotipo grupal de la muestra estudiada presentó una clasificación de mesomorfobalanceado (2,25-5,32-2,26), siendo los defensas los con mayor predominio de este componente. La posición con menor dispersión en su somatotipo son defensas y arqueros, mientras que volantes y delanteros presentan mayor variabilidad. En conclusión, a pesar de existir un somatotipo en común, las diferencias en las varianzas entre posiciones de juego entregan información sobre la relevancia de la optimización morfológica en una posición específica de juego.
\end{abstract}

PALABRAS CLAVE: Somatotipo; Antropometría; Fútbol masculino.

\section{INTRODUCCIÓN}

La valoración de la estructura corporal a partir de variables antropométricas es una parte importante en la evaluación integral de un deportista (Norton \& Olds, 1996). El concepto de optimización morfológica hace referencia a que cada especialidad o modalidad deportiva posee un patrón cineantropométrico específico, que se considera idóneo para alcanzar el éxito (Norton \& Olds, 2001).

El fútbol se ha convertido en el deporte más popular del mundo (COI, 2012), donde la información científica que lo sustenta se ha incrementado notablemente durante las últimas décadas. Un hecho característico de los deportes de equipo, es la heterogeneidad en la demanda física de cada posición de juego, lo que podría llevar a la existencia de diferencias fisiológicas entre los deportistas dependiendo de su posición en el campo (Bangsbo, 2006).

Las características morfológicas del jugador podrían influir en las tácticas de juego del equipo. Cuando se prepara a un deportista se debe identificar en qué medida es necesario que este posea características morfológicas específicas para desempeñar cierta posición de juego (Casajús, 2001;
Gil et al., 2007; Hazir, 2010). En esta línea, parece haber consenso que la forma corporal del futbolista profesional corresponde a mesomorfo-balanceado (Reilly, 1994), sin embargo, las diferencias por posición de juego parecen estar menos claras.

Este estudio pretende describir el somatotipo del futbolista profesional chileno, y determinar la variabilidad existente en el somatotipo por posición de juego.

\section{MATERIAL Y MÉTODO}

Sujetos. Una muestra de 100 jugadores profesionales varo-

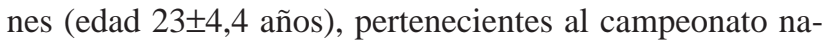
cional de fútbol chileno fueron incluidos en el estudio. Los sujetos fueron agrupados por posición de juego, utilizando la clasificación de estudios previos (Zúñiga \& de León, 2007; Hazir): arqueros $(n=11)$, defensas centrales $(n=17)$, defensas laterales $(n=14)$, medios defensivos $(n=18)$, medios ofensivos $(n=17)$ y delanteros $(n=23)$. Las evaluaciones se reali-

* Unidad de Fisiología del Ejercicio, Laboratorio de Ciencias del Deporte Clínica MEDS.

** Laboratorio de Fisiología, Departamento de Ciencias Biológicas, Facultad de Ciencias Biológicas, Universidad Andrés Bello, Chile.

** Departamento de Deporte y Recreación, Facultad de Ciencias de la Actividad Física. Universidad de Playa Ancha, Chile.

*** Departamento Ciencias de la Actividad Física, Universidad de Los Lagos, Chile.

***** Escuela de Kinesiología, Facultad de Medicina, Universidad Diego Portales, Chile. 
zaron al comienzo de temporada de dos años consecutivos (2010-2011). Todos los participantes dieron su consentimiento informado y los procedimientos fueron realizados de acuerdo a la declaración de Helsinki.

Mediciones. Durante la mañana (9:00-11:00 am) se realizó la medición de 10 variables antropométricas según las normas de la International Society for the Advancement of Kinanthropometry (Marfell-Jones et al., 2006). Se midió talla, masa corporal, 4 pliegues cutáneos (tríceps, subescapular, supraespinal y pierna), 2 diámetros óseos (biepicondilar húmero y fémur) y 2 perímetros musculares (brazo flexionado en tensión y pantorrilla). El peso corporal fue evaluado con balanza digital (Tanita TBF 401A, Japan) y variables antropométricas con el kit de herramientas Health $\&$ Performance ${ }^{\circledR}$.

Análisis de los datos: Se utilizó estadística descriptiva (promedio, desviación estándar, valor mínimo, mediana, máximo) de las variables de estudio, además el análisis de varianza fue aplicado a las características antropométricas básicas.

La media actitudinal del somatotipo (SAM, por su siglas en inglés) y la distancia de dispersión del somatotipo (SDI) fueron calculados para cada posición de juego, de acuerdo a las ecuaciones propuestas por Carter para determinar la homogeneidad de la muestra (Carter \& Honeyman, 1990; Carter, 2002).

Un análisis especial de varianza llamado SANOVA, se utilizó para examinar las diferencias en el somatotipo (distribución tridimensional del somatotipo) de acuerdo a la posición de juego.

El somatotipo fue procesado en el software Somatotype Calculation and Analysis Software versión 1.1 (San Diego, CA, USA), con el que se obtuvieron los somatotipos medios según posición de juego y se realizó un análisis de varianza unidireccional para cada componente. El análisis estadístico fue realizado utilizando el software estadístico Graph pad 5.0 (Graphpad Software, San Diego, CA, USA).

\section{RESULTADOS}

En la Tabla I se describen las características de los sujetos estudiados por posición de juego. No se observaron diferencias significativas en la edad o coeficiente altura-peso, pero de acuerdo al análisis de varianza (ANOVA) se registraron diferencias significativas entre los grupos en la estatura y peso corporal $(\mathrm{p}<0,05)$, siendo los arqueros más altos y pesados vs. otras posiciones de juego.

La media del somatotipo de la muestra estudiada presentó una clasificación de mesomorfo-balanceado $(2,25-$ $5,32-2,26)$. En la figura 1 se muestra la dispersión de los somatopuntos de los sujetos de estudio por posición de juego. El 97\% de los sujetos presenta mesomorfismo como componente principal, distribuidos de manera heterogénea en esa zona de la somatocarta.

\section{MESOMORFISMO}

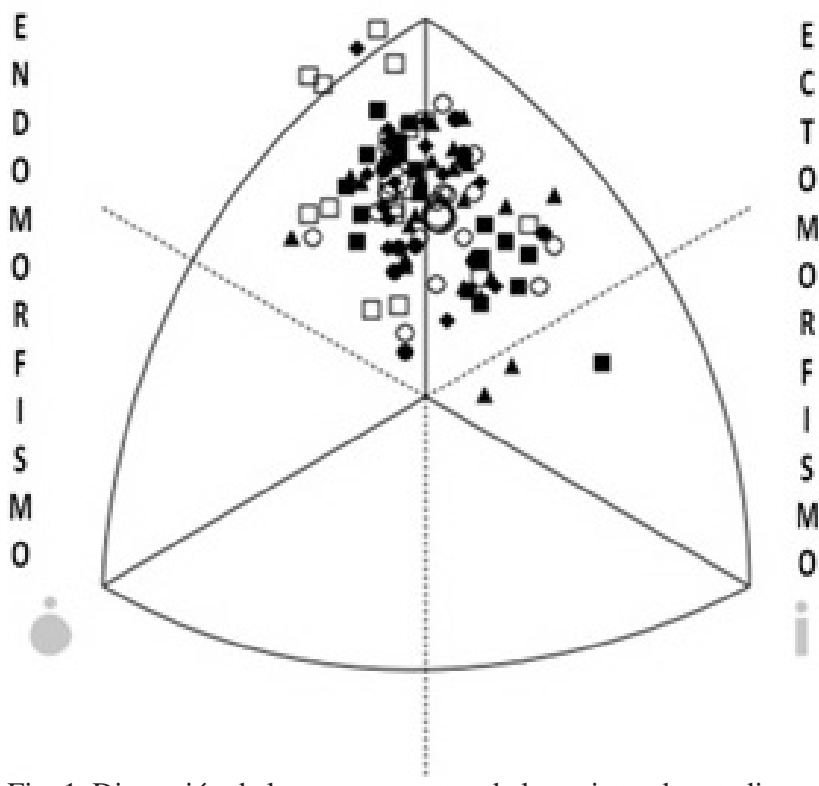

Fig. 1. Dispersión de los somatopuntos de los sujetos de estudio por posición de juego. Arqueros $(\bullet)$, def. centrales (o), def. laterales $(\bullet)$,

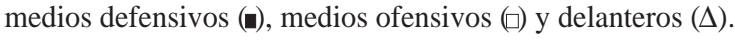

Tabla I. Características antropométricas básicas de la muestra.

\begin{tabular}{lcccccc}
\hline & Arqueros & $\begin{array}{c}\text { Defensas } \\
\text { centrales } \\
(n=17)\end{array}$ & $\begin{array}{c}\text { Defensas } \\
\text { laterales } \\
(n=14)\end{array}$ & $\begin{array}{c}\text { Medios } \\
\text { defensivos } \\
(n=18)\end{array}$ & $\begin{array}{c}\text { Medios } \\
\text { ofensivos } \\
(n=17)\end{array}$ & Delanteros \\
\hline Edad (años) & $23 \pm 4,4$ & $24,8 \pm 4$ & $23,2 \pm 2,6$ & $23,8 \pm 4,9$ & $23,3 \pm 3,8$ & $23,24 \pm 4,5$ \\
Peso (Kg) & $81,3 \pm, 5^{*}$ & $78,2 \pm 4,5$ & $73,03 \pm 5,7$ & $71,61 \pm 6$ & $72,28 \pm 5,4$ & $73,7 \pm 7,1$ \\
Estatura (cm) & $184 \pm 2,8$ & $181,4 \pm 4,2$ & $174,6 \pm 5,7 * \$$ & $175,6 \pm 5,8 *$ & $172,4 \pm 5,2 * \$ \&$ & $177,1 \pm 5,4$ \\
Coef Al-peso & $42,6 \pm 0,60$ & $42,4 \pm 0,7$ & $41,8 \pm 0,7$ & $42,3 \pm 1,1$ & $41,4 \pm 1,0$ & $42,2 \pm 0,9$ \\
\hline
\end{tabular}

* Denota diferencias significativas $(\mathrm{p}<0,05)$ menor vs. arqueros; $\$$ significativamente $(\mathrm{p}<0,05)$ menor vs. defensas centrales; \& significativamente $(\mathrm{p}<0,05)$ menor vs. delanteros. ANOVA, una cola, no pareada. 
En la Tabla II se muestran los componentes del somatotipo según posición de juego. Destaca la homogeneidad, o baja dispersión, en relación al componente de endomorfismo, indicando valores de masa adiposa relativa similares en distintos puestos de juego. Interesantemente, solo los medios ofensivos se clasificaron como endomesomorfos (2,36-5,51-1,76); el resto de las posiciones de juego se clasificaron en mesomorfos-balanceados. La variable con mayor heterogeneidad está representada en el componente ectomórfico, donde la prueba de ANOVA mostró diferencia significativa $(p<0,05)$ entre medios ofensivos y el resto de las posiciones de juego.

En relación a la dispersión de los somatopuntos por posición de juego, la figura 2 muestra que defensas centrales tienen una mayor distribución en el área de mesomorfobalanceado (41\%), a diferencia de los arqueros y medios ofensivos que presentan valores más bajos en esta zona
(18\%). Con respecto a la tendencia a la meso-endomorfia, son los medios ofensivos lo que registran una mayor presencia en ese sector de la somatocarta, que se suma al valor de endomorfismo relativamente elevado $(2,36 \pm 0,67)$ observado en la Tabla II. Por otro lado, los arqueros y los medios defensivos muestran mayor tendencia a la meso-ectomorfia (45\% y $39 \%$, respectivamente).

La figura 3 muestra los índices de dispersión y somatotipos medios según posición de juego. Los valores de SDI observados en las seis posiciones estudiadas fueron >2,0 lo cual indica que existe una gran dispersión de los sujetos en relación a su somatotipo medio. Otra estrategia utilizada para describir la homogeneidad del somatotipo de manera grupal es el SAM (mientras más se acerque a cero este valor, más homogénea es la muestra). En la muestra de estudio ninguna posición reportó valores cercanos a cero.

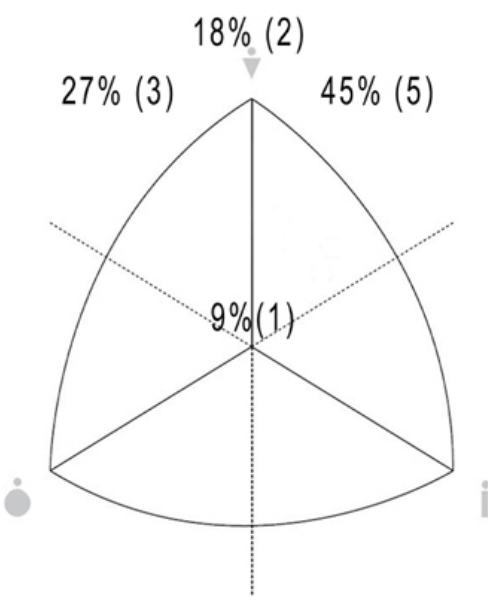

Arqueros

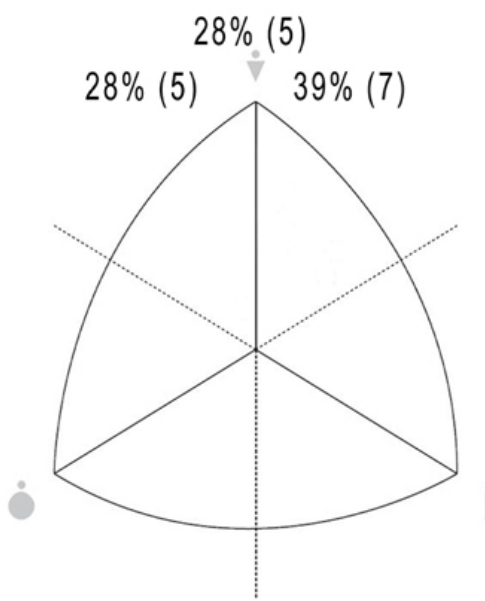

Med. Defensivos

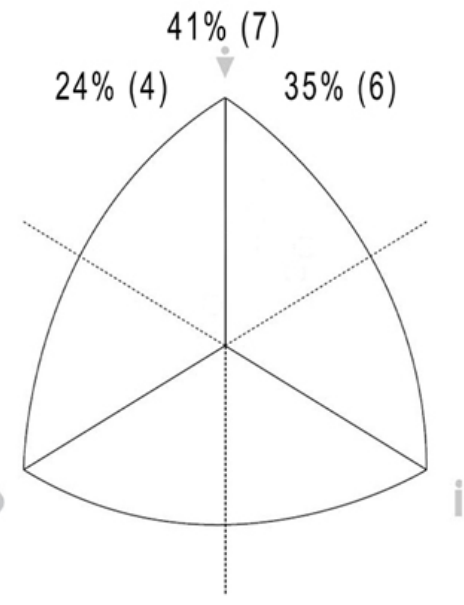

Def. Centrales

$18 \%(3)$

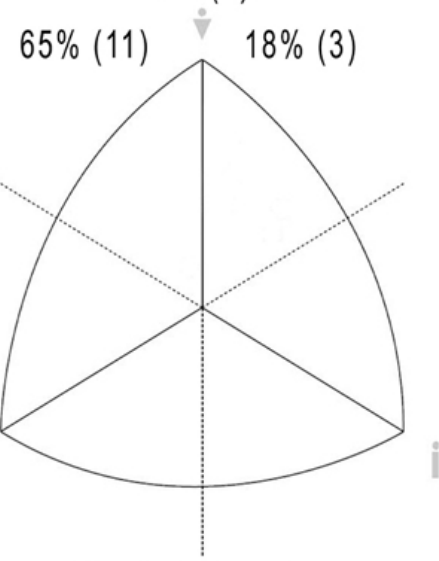

Med. Ofensivos

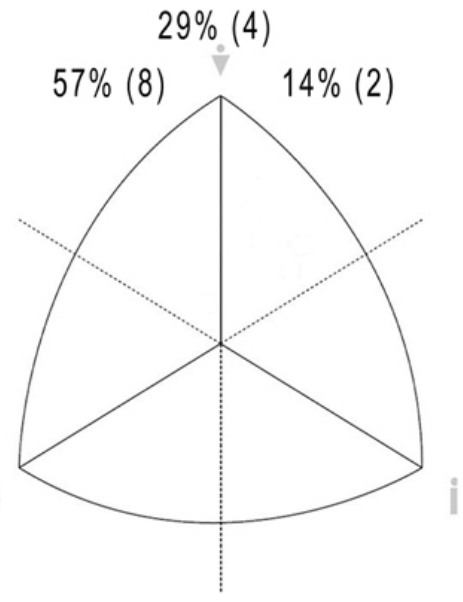

Def. Laterales

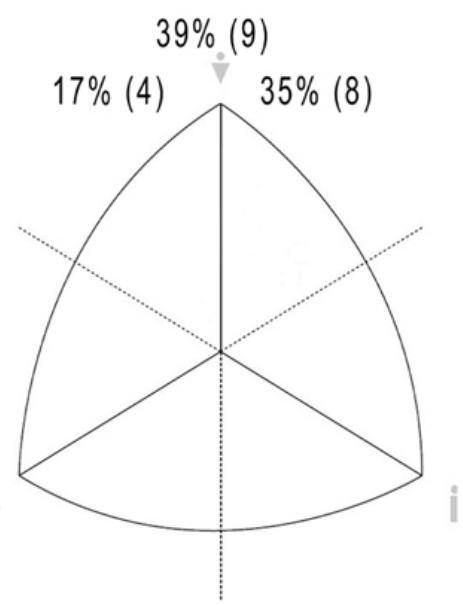

Delanteros

Fig. 2. Dispersión de los somatopuntos de los sujetos de estudio por posición de juego. 
HENRíQUEZ-OLGUíN, C.; BÁEZ, E.; RAMíREZ-CAMPILLO, R. \& CAÑAS, R. Perfil somatotípico del futbolista profesional chileno. Int. J. Morphol., 31(1):225-230, 2013.

Tabla II. Características somatotipicas de futbolistas profesionales Chilenos según posición de juego.

\begin{tabular}{lcccccc}
\hline & Arqueros & $\begin{array}{c}\text { Defensas } \\
\text { centrales } \\
(n=17)\end{array}$ & $\begin{array}{c}\text { Defensas } \\
\text { laterales } \\
(n=14)\end{array}$ & $\begin{array}{c}\text { Medios } \\
\text { defensivos } \\
(n=18)\end{array}$ & $\begin{array}{c}\text { Medios } \\
\text { ofensivos } \\
(n=17)\end{array}$ & $\begin{array}{c}\text { Delanteros } \\
(n=23)\end{array}$ \\
\hline Endomorfismo & $2,35 \pm 0,79$ & $2,23 \pm 0,74$ & $2,27 \pm 0,49$ & $2,16 \pm 0,59$ & $2,36 \pm 0,67$ & $2,14 \pm 0,67$ \\
$\begin{array}{l}\text { Mesomorfismo } \\
\text { Ectomorfismo }\end{array}$ & $5,31 \pm 0,85$ & $5,29 \pm 0,68$ & $5,52 \pm 0,99$ & $5,14 \pm 0,93$ & $5,51 \pm 1,07$ & $5,12 \pm 0,79$ \\
$\begin{array}{l}\text { Clasificación } \\
\text { del somatotipo }\end{array}$ & Meso-balanceado & Meso-balanceado & Meso-balanceado & Meso-balanceado & Endo-mesomorfo Meso-balanceado \\
\hline * Diferencias significativas con las otras posiciones de juego $\mathrm{p}<0,05$. SANOVA test. & & & & &
\end{tabular}

* Diferencias significativas con las otras posiciones de juego $\mathrm{p}<0,05$. SANOVA test.

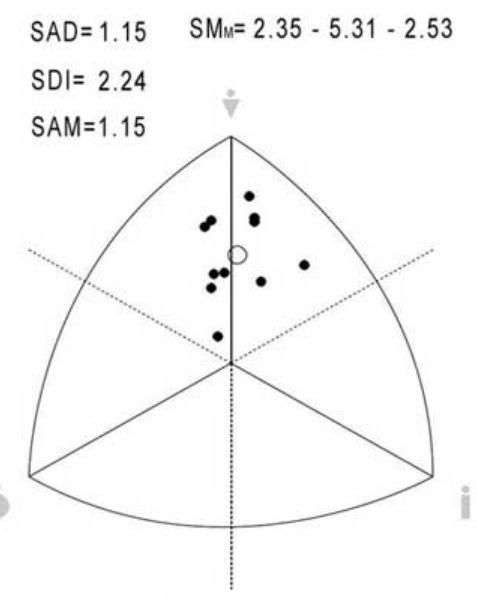

Arqueros

$S A D=1.2 \quad S M=2.16 \cdot 5.14 \cdot 2.42$

$S D I=2.73$

$\mathrm{SAM}=1.63$

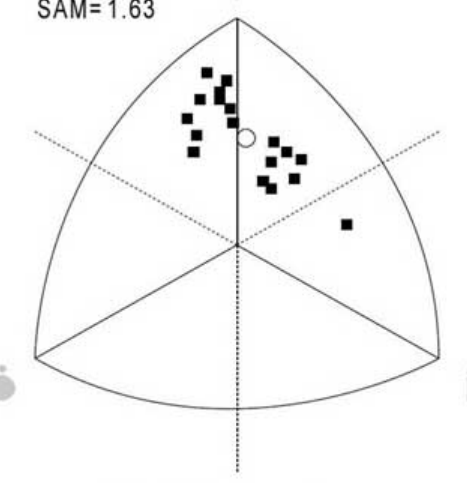

Med.Defensivos
$S A D=1.02 \quad S M M=2.23 \cdot 5.29 \cdot 2.48$

$S D I=2.24$

$S A M=1.02$

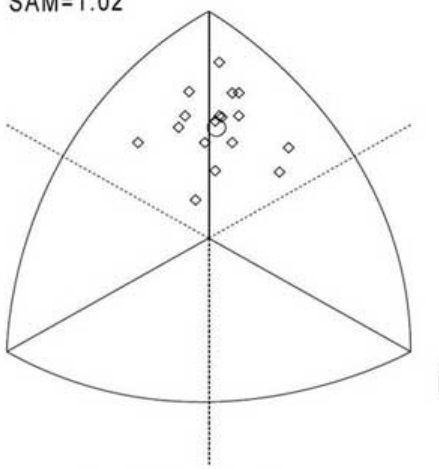

Def.Centrales

$S A D=1.28 \quad S M_{M}=2.36 \cdot 5.51-1.76$

$S D I=2.86$

$S A M=1.28$

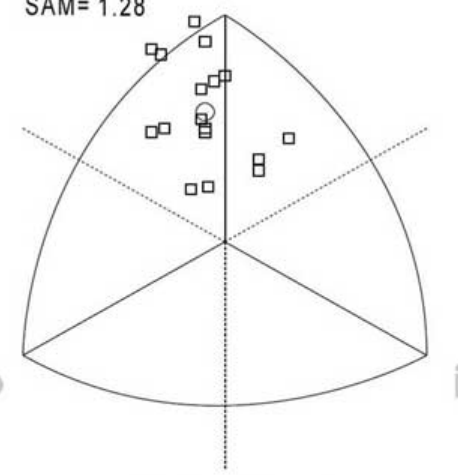

Med. Ofensivos
$S A D=1.06 \quad S M M=2.27-5.52-2.01$

$S D I=2.02$

$S A M=1.06$

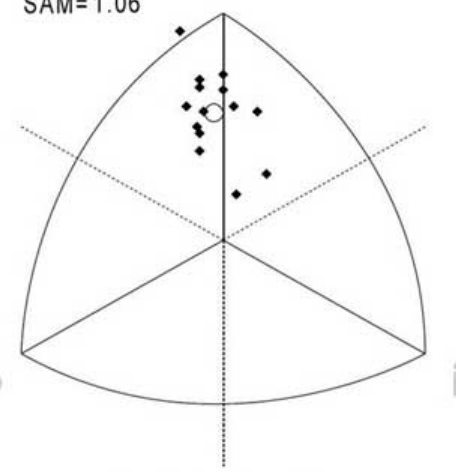

Def. Laterales

$S A D=1.11 \quad S M_{M}=2.14-5.12-2.39$

$\mathrm{SDI}=2.53$

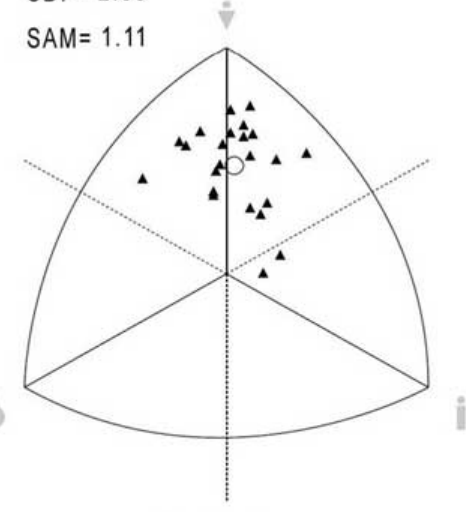

Delanteros

Fig. 3. Índices de dispersión y somatotipos medios según posición de juego. (SMm= Somatotipo medio, SAM= Media actitudinal del somatotipo, SDI= distancia de dispersión del somatotipo).

\section{DISCUSIÓN}

Estudios previos muestran una clara diferencia de peso y talla según posición de juego (Bloomfield et al., 2005).
Este estudio confirmó lo observado previamente, en relación a que los arqueros presentan una talla mayor que el 
resto de los jugadores (Gil et al.), mientras que los medios ofensivos son los sujetos con menor estatura. A pesar de esto, cuando se analiza el coeficiente altura-peso, los sujetos presentaron homogeneidad, lo que indica que la relación entre estatura y peso corporal es similar en el jugador de fútbol, independiente de su posición de juego, concordando con reportes previos que utilizaron el índice de masa corporal en jugadores de distintas posiciones (Hazir).

El presente estudio confirma que el somatotipo del futbolista de elite tiene una estructura morfológica promedio común, reforzando la clasificación de mesomorfo-balanceado observada en futbolistas europeos (Zúñiga \& de León), sudamericanos (Rienzi et al., 2000), africanos y asiáticos (Reilly). Sin embargo, pocos estudios han puesto atención en la variabilidad de esta medida. Los resultados de este estudio muestran que a pesar de tener una media mesomorfo-balanceado, solo el $41 \%$ de los defensas centrales se clasifican como tal, presentándose cifras incluso menores en otras posiciones de juego (Fig. 2).

Por ello, a pesar de existir variados estudios científicos sobre estructura corporal en futbolistas profesionales, la optimización morfológica por posición de juego parece estar menos investigada. Algunos estudios no han mostrado diferencias significativas en distintos puestos de juego (Zúñiga \& de León); estos estudios han utilizado el somatopunto medio (Rienzi) o la división por componentes (Zúñiga \& de León) para realizar dicho análisis.

En el ámbito del rendimiento deportivo, el uso de las medidas de tendencia central para describir características involucradas en el rendimiento puede llevar a conclusiones erróneas o pérdida de valiosa información (Currell \& Jeukendrup, 2008). Considerando al deporte de competición un fenómeno Darwiniano (Puthucheary et al., 2011), las medidas de dispersión nos entregan una visión de qué tan necesarias son ciertas variables para participar en competición. En la presente investigación, utilizamos dos medidas de dispersión (SDI y SAM), propuestas para valorar el somatotipo, observando que cada posición de juego posee distinta dispersión.

Es interesante observar que defensas y arqueros quienes poseen valores menores de SDI, sugiriendo que para desarrollar esa labor en el campo se precisa de una morfología particular, dando luces importantes para la selección de talentos en fútbol. La posición de juego más heterogénea entorno a su somatotipo medio corresponde a los medios ofensivos, lo que podría estar dado por la naturaleza de juego, donde las variables más importantes son la circulación del balón y la entrega del balón en posiciones ventajosas, por sobre la potencia física.
Otros estudios han demostrado que jugadores de mayor nivel tienen valores menores de SDI (Hazir), y a pesar de la variabilidad existente, estas son menores a las observadas en otros deportes de equipo como el hockey o deportes individuales como el atletismo (Casajús \& Aragonés, 1991).

Los resultados de este estudio esclarecen lo documentado en estudios previos, acercándolo a la población chilena, indicando que las diferencias en la distribución del somatotipo de acuerdo a la posición en el juego efectivamente son observadas en futbolistas de alto nivel (Reilly et al., 2000; Casajús), entregando valiosa información para el control integral del futbolista profesional y la selección de talentos.

\section{CONCLUSIONES}

La estructura del somatotipo del futbolista chileno parece estar de acuerdo a la reportada en otras poblaciones. La media del somatotipo corresponde a mesormorfo-balanceado en la mayoría de las posiciones de juego. A pesar de esta aparente homogeneidad, cada posición de juego posee distinta dispersión en relación a su somatotipo medio, la cual debe ser considerada para procesos de selección.

El presente estudio demuestra la importancia de la forma corporal en futbolistas de elite y entrega herramientas profundas de análisis del somatotipo.

AGRADECIMIENTOS. Al Lic. Daniel Zapata por su valioso apoyo en las mediciones antropométricas.

HENRÍQUEZ-OLGUÍN, C.; BÁEZ, E.; RAMÍREZ-CAMPILLO, R. \& CAÑAS, R. Somatotype profile of professional male soccer chilean players. Int. J. Morphol., 31(1):225-230, 2013.

SUMMARY: The aim of this study was to describe the somatotype of Chilean professional soccer players, and to determine the variability in the somatotype by playing position. One hundred professional male players (age 23 \pm 4.4 ) participants in the Chilean national soccer championship were included in the study. We performed the measurement of 10 anthropometric variables according to the rules of the International Society for the Advancement of Kinanthropometry (ISAK). We calculated the 3 components of the Heath-Carter somatotype for each playing position. The somatotype of the sample group presents a balanced mesomorph classification (2.25-5.32-2.26),especially among defenders. The positions with less dispersion in their somatotype were the defenses and goalkeepers, while the flyers and forwards 
show more variability. In conclusion, although there is a common somatotype, differences in the variances between playing positions provide information about the relevance of morphological optimization in a playing position.

KEY WORDS: Anthropometry; Somatotype; Male soccer.

\section{REFERENCIAS BIBLIOGRÁFICAS}

Bangsbo, J. Entrenamiento de la condición física en el fútbol. Barcelona, Paidotribo, 2006.

Bloomfield, J.; Polman, R.; Butterly, R. \& O'Donoghue, P. Analysis of age, stature, body mass, BMI and quality of elite soccer players from 4 European Leagues. J. Sports Med. Phys. Fitness, 45(1):58-67, 2005.

Carter, J. The Heath-Carter anthropometric somatotype. Instruction manual. San Diego, CA, San Diego State University, 2002.

Carter, J. E. \& Honeyman, B. Somaotyping. Development and Applications. Cambridge, UK, Cambridge University Press, 1990.

Casajús, J. A. Seasonal variation in fitness variables in professional soccer players. J. Sports Med. Phys. Fitness, 41(4):463-9, 2001.

Casajús, J. A. \& Aragonés, M. T. Estudio morfológico del futbolista de alto nivel. Composición corporal y somatotipo (Parte 1). Arch. Med. Deporte 8(30):147-51, 1991.

Comité Olímpico Internacional (COI). Official website of the Olympic Movement, 2012. Disponible en: http:// www.olympic.org

Currell, K. \& Jeukendrup, A.E. Validity, reliability and sensitivity of measures of sporting performance. Sports Med., 38(4):297316,2008 .

Gil, S. M.; Gil, J.; Ruiz, F.; Irazusta, A. \& Irazusta, J. Physiological and anthropometric characteristics of young soccer players according to their playing position: relevance for the selection process. J. Strength Cond. Res., 21(2):438-45, 2007.

Hazir, T. Physical characteristics and somatotype of soccer players according to playing level and position. J. Hum. Kinetics, 26: 83-95, 2010.

Marfell-Jones, M. J.; Olds, T.; Stewart, A. D. \& Carter, L. International standards for anthropometric assessment. Potchefstroom, South Africa, International Society for the Advancement of Kinanthropometry (ISAK), 2006.
Norton, K. \& Olds, T. Morphological evolution of athletes over the 20th century: causes and consequences. Sports Med., 31(11):763-83, 2001.

Norton, K. \& Olds, T. Antropometrica. Sydney, University of New Sawth Wales Press, 1996.

Puthucheary, Z.; Skipworth, J. R.; Rawal, J.; Loosemore, M.; Van Someren, K. \& Montgomery, H. E. Genetic influences in sport and physical performance. Sports Med., 41(10):845-59, 2011.

Reilly, T. Physiological profile of the player. En: Ekblom, B. Football: Olympic Handbook of Sports Medicine. London, Blackwell Scientific Publications, 1994. pp.78-94.

Reilly, T.; Bangsbo, J. \& Franks, A. Anthropometric and physiological predispositions for elite soccer. J. Sports Sci., 18(9):669-83, 2000.

Rienzi, E.; Drust, B.; Reilly,T.; Carter, J. E. \& Martin, A. Investigation of anthropometric and work-rate profiles of elite South American international soccer players. J. Sports Med. Phys. Fitnees, 40(2):162-9, 2000.

Zúñiga, G. U. \& de León, F. L. Somatotipo en futbolistas semiprofesionales clasificados por su posición de juego. Rev. Int. Cienc. Deporte, 3(9):29-36, 2007.

Dirección para correspondencia:

Rodrigo Cañas Jamett

Departamento Ciencias Biológicas

Facultad de Ciencias Biológicas

Universidad Andrés Bello

CHILE

Email: rcanas@unab.cl

Recibido : 29-04-2012

Aceptado: 23-11-2012 